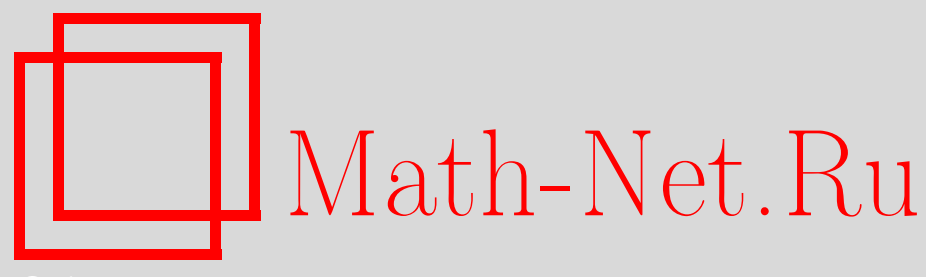

А. Ю. Морозов, Гамильтонов формализм в теориях со старшими производными, ТМФ, 2008, том 157, номер 2, 208-216

DOI: https://doi.org/10.4213/tmf6275

Использование Общероссийского математического портала Math-Net.Ru подразумевает, что вы прочитали и согласны с пользовательским соглашением http://www.mathnet.ru/rus/agreement

Параметры загрузки:

IP : 54.196 .121 .252

26 апреля 2023 г., 12:57:12

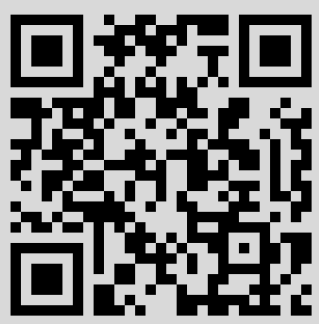




\title{
ГАМИЛЬТОНОВ ФОРМАЛИЗМ В ТЕОРИЯХ СО СТАРШИМИ ПРОИЗВОДНЫМИ
}

\begin{abstract}
Дан краткий обзор основных формул гамильтонова формализма классической механики в случае, когда лагранжиан содержит $N$-е производные по времени от $n$ координат; для нелокальных моделей $N=\infty$.
\end{abstract}

Ключевые слова: теории с высшими производными, гамильтоновы механики, репараметризационная инвариантность.

\section{1. ВВЕДЕНИЕ}

В течение долгого времени теории со старшими производными находились на периферии теоретических исследований. Главная причина этого состоит в том, что старшие производные не слишком часто возникают при описании обычных физических систем. Этому, в свою очередь, можно дать простое объяснение: если наблюдаемый мир описывается низкоэнергетическим пределом некоторой пока неизвестной фундаментальной теории, тогда он с необходимостью управляется лагранжевой динамикой с наинизшим возможным числом производных по времени, и закон Ньютона включает лишь ускорения. С этой точки зрения в фундаменталъной теории не может быть никакого ограничения на число производных, и в самом деле, члены со старшими производными присутствуют в большинстве подходов, от квантово-полевых формулировок теории струн и М-теории [1] до чисто полевых моделей типа асимптотически устойчивой гравитации [2] или квантовой версии недавней модели " $E_{8}$ объединения" [3] в теории частиц. Разумеется, разнообразные нелокальные и бесчисленные некоммутативные модели также попадают в категорию теорий с высшими производными. Более того, даже самые обычные физические теории, скажем классическая электродинамика, на самом деле оказываются несовместными без введения старших производных, если появляется необходимость учитывать радиационные явления и не ограничиваться при этом четырьмя измерениями пространства-времени. Это приходится делать, например, в забавных моделях

* Институт теоретической и экспериментальной физики, Москва, Россия. E-mail: morozov@itep.ru 
ТэВ-ной гравитации [4]. В этих обстоятельствах для решения “проблем" электромагнитной массы и радиационного трения требуется включение в затравочное ("фундаментальное”) действие контрчленов, которые не сводятся лишь к перенормировке массы (перенормировки оказывается достаточно лишь при $d=4$ ), но неизбежно включают также старшие производные по времени [5]. Конечно, старшие производные широко используются и в технических целях, даже там, где введение подобных членов не диктуется физической необходимостью, например для формальной ультрафиолетовой регуляризации теорий с калибровочной или суперсимметриями [6]. Наконец, члены со старшими производными являются общим местом при обсуждении любых эффективных моделей в любой области теории, от физики твердого тела до квантовой гравитации.

По всем этим причинам динамика со старшими производными начинает постепенно привлекать внимание теоретиков, см. относительно недавние обсуждения с различных точек зрения в работах [7]-[19], где развиваются идеи, изложенные в классических статьях, монографиях и учебниках [20]-[37]. Нельзя не отметить, что эта практически нетронутая область очень привлекательна с точки зрения "теоретической теории" и, среди прочего, тесно связана с современным квантово-полевым подходом к топологии [38], $L_{\infty}^{(n)}$-структурам в духе работы [39], нелинейной алгебpe [40] и другим классическим областям фундаментальной науки. Еще в статье [41] было продемонстрировано, что следует ожидать красивых и необычных теоретических явлений при наложении на такие модели условий репараметризационной инвариатности (что, кстати, необходимо во многих мыслимых приложениях). Особенный интерес представляет стоящая за этими моделями новая симплектическая геометрия [42], [43].

Настоящая краткая заметка представляет лишь нулевую главу в исследовании теорий с высшими производными. Здесь приводится краткий список основных элементарных формул, хорошо известных узкому кругу интересующихся ученых еще со времен Остроградского [20]. Эти формулы описывают классическую лагранжеву и гамильтонову динамику, и их необходимо знать для сравнения с результатами, получающимися в разнообразных изощренных подходах, используемых в современной литературе.

\section{2. ЛАГРАЖЕВ ФОРМАЛИЗМ}

Рассмотрим классическую механику с действием

$$
S\left\{q^{\alpha}(t)\right\}=\int L d t
$$

где лагранжиан $L\left(q^{\alpha}, d_{t} q^{\alpha}, \ldots, d_{t}^{N} q^{\alpha}\right)$ зависит от первых $N$ производных по времени $q_{i}^{\alpha} \equiv d_{t}^{i} q^{\alpha}$ от $n$ координатных переменных $q^{\alpha}=q_{0}^{\alpha}, \alpha=1, \ldots, n$. В этих обозначениях

$$
\dot{q}_{i}^{\alpha}=q_{i+1}^{\alpha} .
$$

Определим теперь вариационные производные по $q_{i}^{\alpha}$ для всех $i \geqslant 0$ :

$$
\delta_{\alpha}^{i-1} \equiv \partial_{\alpha}^{i}-d_{t} \partial_{\alpha}^{i+1}+d_{t}^{2} \partial_{\alpha}^{i+2}-\cdots,
$$


где $\partial_{\alpha}^{i}=\partial / \partial q_{i}^{\alpha}$, а импульсы $\Pi_{\alpha}^{i}=\delta_{\alpha}^{i} L$. Операторы вариационного дифференцирования связаны производными по времени:

$$
\dot{\delta}_{\alpha}^{i-1} \equiv d_{t} \delta_{\alpha}^{i-1}=\partial_{\alpha}^{i}-\delta_{\alpha}^{i},
$$

эти соотношения в определенном смысле дуальны (2).

Уравнения движения Эйлера-Лагранжа имеют вид

$$
\Pi_{\alpha}^{-1} \equiv \delta_{\alpha}^{-1} L=0
$$

Они имеют порядок $2 N$ по временнь́м производным. Соответственно, начальные условия налагаются на $q^{\alpha}, d_{t} q^{\alpha}, \ldots, d_{t}^{2 N-1} q^{\alpha}$.

Отметим, что в принятых здесь обозначениях верхние индексы - это не степени. В дальнейшем мы будем пропускать индексы $\alpha$ в некоторых формулах.

\section{3. ГАМИЛЬТОНОВ ФОРМАЛИЗМ}

Фазовое пространство имеет $2 N n$ измерений с канонически сопряженными координатами $q_{i}^{\alpha}$ и $\Pi_{\alpha}^{i}$, где $i=0, \ldots, N-1$. Производные $q_{N}^{\alpha}$ являются заданными функциями от этих независимых переменных.

Замкнутая симплектическая 2-форма

$$
\Omega=d \theta=\sum_{i=0}^{N-1} d \Pi_{\alpha}^{i} \wedge d q_{i}^{\alpha}
$$

сохраняется, $\dot{\Omega}=d_{t} \Omega=0$, в то время как производная по времени от соответствующей пресимплектической 1-формы

$$
\theta=\sum_{i=0}^{N-1} \Pi_{\alpha}^{i} d q_{i}^{\alpha}
$$

точна, $\dot{\theta}=d L$. Уравнения Гамильтона имеют вид

$$
\dot{q}_{i}^{\alpha}=q_{i+1}^{\alpha}=\frac{\partial H}{\partial \Pi_{\alpha}^{i}}, \quad \dot{\Pi}_{\alpha}^{i}=-\Pi_{\alpha}^{i-1}-\frac{\partial L}{\partial q_{i}^{\alpha}}=-\frac{\partial H}{\partial q_{i}^{\alpha}},
$$

где гамильтониан

$$
H(\Pi, q)=\sum_{i=0}^{N-1} \Pi_{\alpha}^{i} q_{i+1}^{\alpha}-L,
$$

а $q_{N}^{\alpha}$ выражены через канонические переменные.

Рассмотрим $\bar{S}(\bar{q}, \overline{\bar{q}})$ - действие $(1)$, вычисленное на классической траектории с граничными условиями $\bar{q}_{i}^{\alpha}=q_{i}^{\alpha}(\bar{t})$ и $\overline{\bar{q}}_{i}^{\alpha}=q_{i}^{\alpha}(\overline{\bar{t}}), i=0, \ldots, N-1$. Тогда

$$
\frac{\partial \bar{S}}{\partial \bar{q}_{i}^{\alpha}}=-\bar{\Pi}_{\alpha}^{i}, \quad \frac{\partial \bar{S}}{\partial \overline{\bar{q}}_{i}^{\alpha}}=\overline{\bar{\Pi}}_{\alpha}^{i} .
$$

Все эти соотношения являются очевидными обобщениями простейшего случая $N=1$ (см. [44]). 


\section{4. ДОКАЗАТЕЛЬСТВА}

Доказательство приведенных выше соотношений не представляет трудности:

$d_{t}\left[\sum_{i=0} d q_{i} \wedge d \delta^{i}\right]=\sum_{i=0}\left(d q_{i+1} \wedge d \delta^{i}-d q_{i} \wedge d \delta^{i-1}+d q_{i} \wedge d \partial^{i}\right)=-d q_{0} \wedge d \delta_{-1}+\sum_{i=0} d q_{i} \wedge d \partial^{i}$.

При действии на $L$ первый член обращается в нуль на уравнениях движения, $\delta^{-1} L=0$, а второй член превращается в

$$
\sum_{i=0} d q_{i} \wedge d\left(\partial_{i} L\right)=\sum_{i, j=0} \frac{\partial^{2} L}{\partial q_{i} \partial q_{j}} d q_{i} \wedge d q_{j}=0
$$

Аналогично

$$
d_{t}\left[\sum_{i=0} d q_{i} \delta^{i}\right]=\sum_{i=0}\left(d q_{i+1} \delta^{i}-d q_{i} \delta^{i-1}+d q_{i} \partial^{i}\right)=-d q_{0} \delta^{-1}+d
$$

И снова при действии на $L$ первый член обращается в нуль на уравнениях движения.

Производные гамильтониана в (6) равны

$$
\begin{aligned}
\frac{\partial H}{\partial \Pi^{i}} & =q_{i+1}+\left(\Pi^{N-1}-\frac{\partial L}{\partial q_{N}}\right) \frac{\partial q_{N}}{\partial \Pi^{i}} \\
-\frac{\partial H}{\partial q_{i}} & =-\Pi^{i-1}\left(1-\delta_{i, 0}\right)+\frac{\partial L}{\partial q_{i}}-\left(\Pi^{N-1}-\frac{\partial L}{\partial q_{N}}\right) \frac{\partial q_{N}}{\partial q_{i}} .
\end{aligned}
$$

Члены в скобках в правой стороне равенств обращаются в нуль, поскольку $\Pi^{N-1}=$ $\delta^{N-1} L=\partial^{N} L$.

Наконец, изменение действия $S\{q(t)\}$ при вариации его аргумента $\delta q(t)$ равно

$$
\delta S=\int \sum_{i=0}^{N} \delta x_{i} \partial^{i} L=\oint \delta x_{j} \delta^{j} L+\int \delta^{-1} L
$$

На классической траектории второй член исчезает, а граничный вклад в первом члене порождает (8).

\section{5. ПУТЬ К КОГОМОЛОГИЧЕСКОЙ ФОРМУЛИРОВКЕ}

Ключевую роль в только что проделанном вычислении играет оператор

$$
\hat{A}=\sum_{i=1}(-1)^{i} \partial^{i-1} \otimes \partial^{-i}=\frac{1}{1 \otimes 1+\partial \otimes \partial^{-1}} 1 \otimes \partial^{-1}=\frac{1}{\partial^{-1} \otimes \partial+1 \otimes 1} \partial^{-1} \otimes 1 .
$$

Он является формальным обращением оператора $\partial$, действующего на произведения по правилу Лейбница:

$$
(\partial \otimes 1+1 \otimes \partial) \hat{A}=\frac{1}{1 \otimes 1+\partial \otimes \partial^{-1}}\left(\partial \otimes \partial^{-1}+1 \otimes 1\right)=1 \otimes 1 .
$$


На концептуальном уровне для $\partial=d_{t}$

$$
\Omega=\hat{A}_{*} d q \hat{\otimes} \frac{\delta L}{\delta q} .
$$

В самом деле, для

$$
\frac{\delta}{\delta q_{i}}=\frac{\partial}{\partial q_{i}}-\partial \frac{\partial}{\partial q_{i+1}}+\cdots
$$

имеем $\partial \delta_{i+1}=\partial_{i}-\delta_{i}$ или

$$
\frac{\delta}{\delta q_{i+1}}=-\partial_{*}^{-1} \frac{\delta}{\delta q_{i}}=\cdots=(-1)^{i} \partial_{*}^{-i} \frac{\delta}{\delta q},
$$

где $\partial_{*}^{-1}$ правильно учитывает $\partial / \partial q_{i}$, лежащие в “когомологии” $\partial$.

Более аккуратный анализ должен учесть различие между $\hat{A}_{*}$ и $\hat{A}$. Именно это различие позволяет $\Omega$ не обращаться тождественно в нуль, несмотря на то что $\delta L=0$. Обращение $\hat{A}_{*}$ в нуль происходит только при взятии производной $\partial$ по времени, до этого мы имеем ненулевое выражение.

\section{6. ПРИМЕРЫ С НЕЛОКАЛЬНОСТЬЮ}

Развитый выше гамильтонов формализм можно немедленно применить к любым функционалам, в том числе нелокальным.

В качестве квадратичного по $q$ примера можно взять

$$
S\{q(t)\}=\int q \frac{1}{1+M^{-2} \partial^{2}} q d t
$$

или

$$
S\{q(t)\}=\int q(t) q(t+\epsilon) d t=\int q(t) e^{\epsilon \partial} q(t) d t
$$

(в этом случае решения уравнений Эйлера-Лагранжа - это любые антипериодические функции $q(\cdot), q(t+2 \epsilon)=-q(t))$, или, в общем случае,

$$
S\{q(t)\}=\frac{1}{2} \int\left(\sum_{n} a_{n}\left(\partial^{n} q\right)^{2}\right) d t,
$$

где $a_{n}$ не зависит от времени (последнее ограничение, естественно, можно и не налагать).

Мы имеем

$$
\Pi_{i}=\delta^{i} L=\sum_{j=0}(-1)^{j} a_{i+j+1} \partial^{i+2 j+1} q
$$

и

$$
\Omega=\sum_{i=0} d q_{i} \wedge d \Pi_{i}=\sum_{i, j \geqslant 0}(-1)^{j} a_{i+j+1} d q_{i} \wedge d q_{i+2 j+1} .
$$

Отметим, что члены $d q_{i} \wedge d q_{i+2 j}$ в этом разложении не появляются. Поскольку коэффициенты $A_{i j}$ в матрице $\Omega=\sum_{i<j} A_{i j} d q_{i} \wedge d q_{j}$ не зависят от времени, они обязаны иметь специальный вид $A_{i j}=(-1)^{j} A_{i+j}$ (хорошо знакомый из теории $\tau$-функций для цепочек Тоды [45]) в силу условий сохранения

$$
\dot{A}_{i j}+A_{i-1, j}+A_{i, j-1}=0 .
$$




\section{7. РЕПАРАМЕТРИЗАЦИОННО-ИНВАРИАНТНЫЕ ДЕЙСТВИЯ}

Рассмотрим преобразования $t \rightarrow u(t)=t+\epsilon(t)$, при этом

$$
\begin{aligned}
& q_{1}^{\alpha} \equiv \dot{q}^{\alpha} \rightarrow u q_{1}^{\alpha} \rightarrow q_{1}^{\alpha}+\epsilon q_{1}^{\alpha}, \\
& q_{2}^{\alpha} \equiv \ddot{q}^{\alpha} \rightarrow u^{2} q_{2}^{\alpha}+u \dot{u} q_{1}^{\alpha} \rightarrow q_{2}^{\alpha}+2 \epsilon q_{2}^{\alpha}+\dot{\epsilon} q_{1}^{\alpha}, \\
& q_{3}^{\alpha} \rightarrow u^{3} q_{3}^{\alpha}+3 u^{2} \dot{u} q_{2}^{\alpha}+u \dot{u}^{2} q_{1}^{\alpha}+u^{2} \ddot{u} q_{1}^{\alpha} \rightarrow q_{3}^{\alpha}+3 \epsilon q_{3}^{\alpha}+3 \dot{\epsilon} q_{2}^{\alpha}+\ddot{\epsilon} q_{1}^{\alpha},
\end{aligned}
$$

или, что эквивалентно,

$$
q_{k}^{\alpha} \equiv \partial_{t}^{k} q^{\alpha} \rightarrow q_{k}^{\alpha}+\sum_{l=0}^{k-1} C_{k}^{k-l-1} q_{k-l}^{\alpha} \partial_{t}^{l} \epsilon
$$

Инвариантность действия означает, что для любого $\epsilon=\epsilon(t)$

$$
\begin{aligned}
\epsilon L=\epsilon & \left\{\dot{q}^{\alpha} \frac{\partial}{\partial \dot{q}^{\alpha}}+2 \ddot{q}^{\alpha} \frac{\partial}{\partial \ddot{q}^{\alpha}}+3 q_{3}^{\alpha} \frac{\partial}{\partial q_{3}^{\alpha}}+4 q_{4}^{\alpha} \frac{\partial}{\partial q_{4}^{\alpha}}+\cdots\right\} L+ \\
& +\dot{\epsilon}\left\{\dot{q}^{\alpha} \frac{\partial}{\partial \ddot{q}^{\alpha}}+3 \ddot{q}^{\alpha} \frac{\partial}{\partial q_{3}^{\alpha}}+6 q_{3}^{\alpha} \frac{\partial}{\partial q_{4}^{\alpha}}+\cdots\right\} L+ \\
& +\ddot{\epsilon}\left\{\dot{q}^{\alpha} \frac{\partial}{\partial q_{3}^{\alpha}}+4 \ddot{q}^{\alpha} \frac{\partial}{\partial q_{4}^{\alpha}}+\cdots\right\} L+ \\
& +\left(\partial_{t}^{3} \epsilon\right)\left\{\dot{q}^{\alpha} \frac{\partial}{\partial q_{4}^{\alpha}}+\cdots\right\} L+\cdots
\end{aligned}
$$

или

$$
\sum_{m=0}^{\infty} K_{m} \partial_{t}^{m} \epsilon=0, \quad \text { т.e. } \quad K_{m}=\sum_{k \geqslant 1}^{\infty} C_{m+k}^{k-1} q_{k}^{\alpha} \frac{\partial L}{\partial q_{k+m}^{\alpha}}-L \delta_{m, 0}=0 \text {. }
$$

В этих формулах $C_{m+k}^{k-1}=\frac{(m+k) !}{(k-1) !(m+1) !}-$ биномиальные коэффициенты.

Как следствие, обычный гамильтониан (7) тождественно равен нулю,

$$
\begin{aligned}
H= & \sum_{i=0}^{N-1} \Pi_{\alpha}^{i} q_{i+1}^{\alpha}-L=\sum_{k \geqslant 1} q_{k}^{\alpha}\left(\frac{\partial L}{\partial q_{k}^{\alpha}}-\frac{d}{d t} \frac{\partial L}{\partial q_{k+1}^{\alpha}}+\frac{d^{2}}{d t^{2}} \frac{\partial}{\partial q_{k+2}^{\alpha}}-\cdots\right)-L= \\
= & K_{0}-\sum_{k \geqslant 1}\left((k-1) q_{k}^{\alpha} \frac{\partial L}{\partial q_{k}^{\alpha}}+q_{k}^{\alpha} \frac{\partial^{2}}{\partial q_{k+1}^{\alpha} \partial q_{l}^{\beta}} q_{l+1}^{\beta}-\cdots\right)= \\
= & K_{0}-\frac{d K_{1}}{d t}+\sum_{k}\left(\frac{(k-1)(k-2)}{2} q_{k}^{\alpha} \frac{\partial L}{\partial q_{k}^{\alpha}}+\frac{(k-1)(k+2)}{2} q_{k}^{\alpha} \frac{\partial^{2} L}{\partial q_{k+1}^{\alpha} \partial q_{l}^{\beta}} q_{l+1}^{\beta}+\right. \\
& \left.\quad+q_{k}^{\alpha} \frac{\partial^{2} L}{\partial q_{k+2}^{\alpha} \partial q_{l}^{\beta}} q_{l+2}^{\beta}+q_{k}^{\alpha} \frac{\partial^{3} L}{\partial q_{k+2}^{\alpha} \partial q_{l}^{\beta} \partial q_{m}^{\gamma}} q_{l+1}^{\beta} q_{m+1}^{\gamma}-\cdots\right)= \\
= & K_{0}-\frac{d K_{1}}{d t}+\frac{d^{2} K_{2}}{d t^{2}}-\frac{d^{3} K_{3}}{d t^{3}}+\cdots=0 .
\end{aligned}
$$


Как и во всякой калибровочно инвариантной теории, в уравнения Гамильтона входит не наивный гамильтониан, а связь $\Phi$ - генератор калибровочного преобразования [27]:

$$
\dot{q}^{\alpha}=\frac{\partial \Phi}{\partial \Pi_{\alpha}}, \quad \dot{\Pi}_{\alpha}=-\frac{\partial \Phi}{\partial q^{\alpha}} .
$$

В статье [41] разбирался специальный случай, в котором отсутствует зависимость от $q_{0}^{\alpha}$ и $N=1$, и уже этот случай оказался неожиданно богатым и интересным.

\section{8. СЛУЧАЙ $N=2$ И $N=2$}

Согласно (23) в этом случае лагранжиан $L$ является функцией от $q_{1}, q_{2}, v_{1}=\dot{q}_{1}$, $v_{2}=\dot{q}_{2}$ и $z=v_{1} a_{2}-v_{2} a_{1}=v_{1} \dot{v}_{2}-v_{2} \dot{v}_{1}$ и имеет определенную степень однородности:

$$
\left.3 z \frac{\partial L}{\partial z}\right|_{v_{1,2}}+\left.v_{1} \frac{\partial L}{\partial v_{1}}\right|_{v_{2}, z}+\left.v_{2} \frac{\partial L}{\partial v_{2}}\right|_{v_{1}, z}=L
$$

Импульсы задаются формулами

$$
\begin{aligned}
\Pi_{1}^{0} & =\left.\frac{\partial L}{\partial v_{1}}\right|_{v_{2}, a_{1,2}}-\left.\frac{d}{d t} \frac{\partial L}{\partial a_{1}}\right|_{v_{1,2}, a_{2}}=\left.\frac{\partial L}{\partial v_{1}}\right|_{v_{2}, z}+\left.a_{2} \frac{\partial L}{\partial z}\right|_{v_{1,2}}+\frac{d}{d t}\left(\left.v_{2} \frac{\partial L}{\partial z}\right|_{v_{1,2}}\right)= \\
& =\left.\frac{\partial L}{\partial v_{1}}\right|_{v_{2}, z}+\left.2 a_{2} \frac{\partial L}{\partial z}\right|_{v_{1,2}}+\left.v_{2} \frac{d}{d t} \frac{\partial L}{\partial z}\right|_{v_{1,2}}= \\
& =\frac{\partial L}{\partial v_{1}}+2 a_{2} \frac{\partial L}{\partial z}+v_{2}\left(v_{1} \frac{\partial^{2} L}{\partial z \partial q_{1}}+a_{1} \frac{\partial^{2} L}{\partial z \partial v_{1}}+\left(v_{1} w_{2}-v_{2} w_{1}\right) \frac{\partial^{2} L}{\partial z^{2}}\right), \\
\Pi_{2}^{0} & =\left.\frac{\partial L}{\partial v_{2}}\right|_{v_{1}, a_{1,2}}-\left.\frac{d}{d t} \frac{\partial L}{\partial a_{2}}\right|_{v_{1,2}, a_{1}}, \\
\Pi_{1}^{1} & =\left.\frac{\partial L}{\partial a_{1}}\right|_{v_{1,2}, a_{2}}, \quad \Pi_{1}^{2}=\left.\frac{\partial L}{\partial a_{2}}\right|_{v_{1,2}, a_{1}} .
\end{aligned}
$$

Не только в этом примере, но и всегда репараметризационная инвариантность равносильна условию однородности функции, зависящей от специфических комбинаций исходных переменных,

$$
z_{i j}=\dot{q}_{i} \ddot{q}_{j}-\ddot{q}_{i} \dot{q}_{j} \sim \partial_{t}\left(\frac{\dot{q}_{i}}{\dot{q}_{j}}\right), \quad z_{i j ; k l} \sim \partial_{t}\left(\frac{\dot{z}_{i j}}{\dot{z}_{k l}}\right), \quad z_{i j ; k l \mid i^{\prime} j^{\prime} ; k^{\prime} l^{\prime}} \sim \partial_{t}\left(\frac{\dot{z}_{i j ; k l}}{\dot{z}_{i^{\prime} j^{\prime} ; k^{\prime} l^{\prime}}}\right)
$$

и т.д., которые являются “элементарными мономами”, однородно преобразующимися под действием репараметризаций времени. Разумеется, эти величины играют существенную роль в дальнейшем развитии теории (см., например, [43]).

Благодарности. Я признателен за многочисленные обсуждения Валерию Долотину и другим участникам его семинаров в ИТЭФ. Я благодарен за гостеприимство под эгидой JSPS Университету г. Осаки, где этот текст был окончательно доработан. Моя работа частично поддерживается Российским Агенством по ядерной энергии (совместный грант 06-01-92059-CE), NWO (проекты № 047.011.2004.026 и ANR-05-BLAN-0029-01), INTAS (грант № 05-1000008-7865), РФФИ (грант № 07-0200645) и Программой поддержки ведущих научных школ (грант № НШ-8004.2006.2). 


\section{Список литературы}

[1] E. Fradkin, A. Tseytlin, Nucl. Phys. B, 261:1 (1985), 1-27; E. Witten, Nucl. Phys. B, 268:2 (1986), 253-294; W. Siegel, Introduction to String Field Theory, Adv. Ser. Math. Phys., 8, World Scientific, Teaneck, NJ, 1988.

[2] M. Reuter, F. Saueressig, Functional renormalization group equations, asymptotic safety and quantum Einstein gravity, arXiv: 0708.1317.

[3] A. Garrett Lisi, An exeptionally simple theory of everything, arXiv: 0711.0770.

[4] N. Arkani-Hamed, S. Dimopoulos, G. Dvali, Phys. Lett. B, 429:3-4 (1998), 263-272; arXiv: hep-ph/9803315; Phys. Today, 55:2 (2002), 35-40; G. Landsberg, Collider searches for extra dimensions, arXiv: hep-ex/0412028; A. Mironov, A. Morozov, T. Tomaras, Can Centauros or Chirons be the first observations of evaporating mini black holes?, arXiv: hep-ph/0311318; Facta Univ. Ser. Phys. Chem. Tech., 4 (2006), 381-404; arXiv: 0710.3395; I. Ya. Aref'eva, I. V. Volovich, Int. J. Geom. Meth. Mod. Phys., 5:4 (2008), 641-651; arXiv: 0710.2696.

[5] B. Kosyakov, Introduction to the Classical Theory of Particles and Fields, Springer, Berlin, 2007; D. V. Gal'tsov, Phys. Rev. D, 66:2 (2002), 025016; arXiv: hep-th/0112110; P. O. Kazinski, S. L. Lyakhovich, A. A. Sharapov, Phys. Rev. D, 66:2 (2002), 025017; arXiv: hep-th/0201046; Yu. Yaremko, SIGMA, 1 (2005), 012; arXiv: math-ph/0511075; П. Казинский, Эффективная динамика сингулярных источников в классической теории поля, дисс. ... канд. ф.-м. наук, Томский гос. ун-т, Томск, 2007; A. Mironov, A. Morozov, On the problem of radiation friction beyond 4 and 6 dimensions, arXiv: 0710.5676; Писвма в ЖЖЭТФ, 85:1 (2007), 9-14; arXiv: hep-ph/0612074; ТМФ, 156:2 (2008), 282-291; arXiv: hep-th/0703097; D. Galakhov, Писъма в ЖЭТФ, 87:8 (2008), 522-527; arXiv: 0710.5688.

[6] A. Slavnov, Nucl. Phys. B, 97:1 (1975), 155-164; A. B. Pimenov, E. S. Shevtsova, A. A. Soloshenko, K. V. Stepanyantz, Higher derivative regularization and quantum corrections in $N=1$ supersymmetric theories, arXiv: 0712.1721.

[7] R. Woodard, Phys. Rev. A, 62:5 (2000), 052105; arXiv: hep-th/0006207.

[8] K. Bering, A note on non-locality and Ostrogradski's construction, arXiv: hep-th/0007192.

[9] R. Miron, D. Hrimiue, H. Shimada, S. V. Sabau, The Geometry of Hamilton and Lagrange Spaces, Fund. Theories Phys., 118, Kluwer, Dordrecht, 2001.

[10] S. Hawking, T. Hertog, Phys. Rev. D, 65:10 (2002), 103515; arXiv: hep-th/0107088.

[11] T.-C. Cheng, P.-M. Ho, M.-C. Yeh, Phys. Rev. D, 66:8 (2002), 085015; arXiv: hep-th/0206077.

[12] J. Gomis, K. Kamimura, T. Ramirez, Nucl. Phys. B, 696:1-2 (2004), 263-291; arXiv: hep-th/0311184.

[13] A. Smilga, Phys. Lett. B, 632:2-3 (2006), 433-438; arXiv: hep-th/0503213; 6D superconformal theory as the theory of everything, arXiv: hep-th/0509022.

[14] M.S. Plyushchay, EJTP, 3:10 (2006), 17-31; arXiv: math-ph/0604022.

[15] I. Cortese, J. A. Garcia, Phys. Lett. A, 358:5-6 (2006), 327-333; arXiv: hep-th/0605156; Int. J. Geom. Methods Mod. Phys., 4:5 (2007), 789-805; arXiv: hep-th/0703205.

[16] S. L. Lyakhovich, A. A. Sharapov, JHEP, 1 (2007), 047; arXiv: hep-th/0612086.

[17] K. Bolonek, P. Kosiński, J. Phys. A, 40:38 (2007), 11561-11567; arXiv: quant-ph/0612091.

[18] C. S. Acatrinei, J. Phys. A, 40:43 (2007), F929-F933; arXiv: 0708.4351.

[19] K. Andrzejewski, J. Gonera, P. Maslanka, A note on the Hamiltonian formalism for higher-derivative theories, arXiv: 0710.2976.

[20] М. В. Остроградский, Полн. собр. трудов. Т. 2, Изд-во АН УССР, Киев, 1961, 139-233.

[21] E. T. Wittaker, A Treatise on the Analytical Dynamics of Particles and Rigid Bodies. With an Introduction to the Problem of Three Bodies, Cambridge University Press, New York, 1988. 
[22] A. Pais, G. Uhlenbeck, Phys. Rev., 79 (1950), 145-165.

[23] П. Дирак, Лекиии по квантовой механике, РХД, Ижевск, 1999.

[24] A. Slavnov, Nucl. Phys. B, 31:2 (1971), 301-315.

[25] А.А. Славнов, Л. Д. Фаддеев, Введение в квантовую теорию калибровочных полей, Наука, М., 1978.

[26] Б. А. Дубровин, С.П. Новиков, А. Т. Фоменко, Современная геометрия: Методъ и приложения. Т. 2. Геометрия и топология многообразий, УРСС, М., 2001.

[27] Д. М. Гитман, И. В. Тютин, Каноническое квантование полей со связями, Наука, М., 1986.

[28] D. Eliezer, R. Woodard, Nucl. Phys. B, 325:2 (1989), 389-469.

[29] M. Plyushchay, Modern Phys. Lett. A, 4:9 (1989), 837-847.

[30] J. M. Pons, Lett. Math. Phys., 17:3 (1989), 181-189.

[31] J. Z. Simon, Phys. Rev. D, 41:12 (1990), 3720-3733.

[32] M. Kreuzer, Classical Quantum Gravity, 7:8 (1990), 1303-1317.

[33] M. Henneaux, C. Teitelboim, Quantization of Gauge Systems, Princeton University Press, Princeton, NJ, 1992.

[34] J. Govaerts, M. S. Rashid, The Hamiltonian formulation of higher order dynamical systems, arXiv: hep-th/9403009.

[35] J. Llosa, J. Vives, J. Math. Phys., 35:6 (1994), 2856-2877.

[36] H.-J. Schmidt, Phys. Rev. D, 49:12 (1994), 6354-6366; Erratum, 54 (1996), 7906; arXiv: gr-qc/9404038.

[37] T. Nakamura, S. Hamamoto, Progr. Theoret. Phys., 95:3 (1996), 469-484; arXiv: hep-th/9511219.

[38] А. Ю. Морозов, УФН, 150 (1986), 337-416; А. Городенцев, А. Лосев, Лекиии на школах в Домбае и Дубне, 2004; D. Krotov, A. Losev, A. Gorodentsev, Quantum field theory as effective $B V$ theory from Chern-Simons, arXiv: hep-th/0603201; P. Mnev, Notes on simplicial BF theory, arXiv: hep-th/0610326.

[39] V. Dolotin, A. Morozov, Sh. Shakirov, Phys. Lett. B, 651:1 (2007), 71-73; arXiv: 0704.2884; В. В. Долотин, А. Ю. Морозов, Ш. Р. Шакиров, ТМФ, 156:1 (2008), 3-37; arXiv: 0704.2609.

[40] I. Gelfand, M. Kapranov, A. Zelevinsky, Discriminants, Resultants and Multidimensional Determinants, Birkhäuser, Boston, MA, 1994; V. Dolotin, A. Morozov, Introduction to Non-Linear Algebra, World Scientific, Hackensack, NJ, 2007; arXiv: hep-th/0609022.

[41] D. Fairlie, J. Govaerts, A. Morozov, Nucl. Phys. B, 373:1 (1992), 214-232; arXiv: hep-th/9110022.

[42] В. Долотин, А. Стояновский, Въступления на семинарах ИТЭФ, 2006-2007.

[43] П. Дунин-Барковский, А. Слепцов, ТМФ, 158:1 (2009), 72-97; arXiv: 0801.4293.

[44] Л. Д. Ландау, Е. М. Лифшиц, Теоретическая физика. Т. І. Механика, Наука, М., 1957.

[45] А. Морозов, УФН, 164:1 (1994), 3-62; arXiv: hep-th/9303139; Matrix models as integrable systems, arXiv: hep-th/9502091. 